\title{
The function of tcf3 in medaka embryos: efficient knockdown with pePNAs
}

\author{
Gerlinde Doenz ${ }^{1 \dagger}$, Sebastian Dorn ${ }^{1 \dagger}$, Narges Aghaallaei ${ }^{2,3}$, Baubak Bajoghli $^{3}$, Elisabeth Riegel ${ }^{1}$, Michaela Aigner $^{4}$, \\ Holger Bock ${ }^{5}$, Birgit Werner ${ }^{6}$, Thomas Lindhorst ${ }^{6}$ and Thomas Czerny ${ }^{1 *}$ (i)
}

\begin{abstract}
Background: The application of antisense molecules, such as morpholino oligonucleotides, is an efficient method of gene inactivation in vivo. We recently introduced phosphonic ester modified peptide nucleic acids (PNA) for in vivo loss-of-function experiments in medaka embryos. Here we tested novel modifications of the PNA backbone to knockdown the medaka tcf3 gene.

Results: A single tcf3 gene exists in the medaka genome and its inactivation strongly affected eye development of the embryos, leading to size reduction and anophthalmia in severe cases. The function of Tcf3 strongly depends on co-repressor interactions. We found interactions with Groucho/Tle proteins to be most important for eye development. Using a dominant negative approach for combined inactivation of all groucho/tle genes also resulted in eye phenotypes, as did interference with three individual tle genes.

Conclusions: Our results show that side chain modified PNAs come close to the knockdown efficiency of morpholino oligonucleotides in vivo. A single medaka tcf3 gene combines the function of the two zebrafish paralogs hdl and tcf3b. In combination with Groucho/Tle corepressor proteins Tcf3 acts in anterior development and is critical for eye formation.
\end{abstract}

Keywords: PNA, Knockdown, Medaka, Tcf3, Groucho, Tle

\section{Background}

Antisense molecules which inhibit mRNA activity through a Watson-Crick base pair mechanism have been studied for several decades. Blocker molecules, such as morpholino oligonucleotides and peptide nucleic acids (PNAs), show high specificity and low toxic effects (reviewed by Summerton [1]). The morpholino backbone consists of 6-membered morpholine rings connected by non-ionic phosphorodiamidate linker-units [2]. In PNAs the phosphate ribose ring of DNA is replaced with repeating $\mathrm{N}$-[2-aminoethyl]-glycine (aeg) units linked by amide bonds. This provides a neutral backbone and results in complementary PNA/DNA strands having stronger binding affinity than DNA/DNA [3]. In addition, introduction of mismatches destabilizes the PNA/ DNA duplex more than they do in DNA/DNA. PNAs are highly stable against enzymatic degradation and changes in

\footnotetext{
* Correspondence: thomas.czerny@fh-campuswien.ac.at

${ }^{\dagger}$ Equal contributors

${ }^{1}$ Department for Applied Life Sciences, University of Applied Sciences, FH

Campus Wien, Helmut-Qualtinger-Gasse 2, A-1030 Vienna, Austria

Full list of author information is available at the end of the article
}

temperature and $\mathrm{pH}$. Moreover, due to their short length (13-18 bases) the likelihood that secondary structures form is reduced which results in stable and sequence specific probes [4-6]. Unmodified aegPNAs, however, show low efficiency in vivo, therefore, various terminal- and internal modifications have been tested. For example, the addition of lysine residues improves their solubility [7]. The introduction of negative charges in mixed sequence HypNA-PNAs allowed specific down regulation of target genes in zebrafish embryos [8]. Recently, pePNAs which contain phosphonic ester (pe) side chains in an otherwise non-modified aegPNA backbone were successfully applied to target six 3 in medaka embryos. In particular combined versions containing both aeg non-modified- and pe-modified PNA blocks were most effective. In a direct comparison morpholino oligonucleotides were active at lower concentrations, but also showed higher toxicity [9].

Tcf/Lef (T-cell factor/lymphoid enhancer factor) proteins belong to the high mobility group (HMG) box-containing family of transcription factors [10]. They are the most distal players of the canonical Wnt 
pathway and the main partner for $\beta$-catenin in gene regulation $[11,12]$. In the absence of Wnt ligands, $\beta$-catenin is degraded in the cytosol and Tcf/Lef interacts with transcriptional co-repressors such as Groucho/Tle (Groucho/ Transducin-like enhancer) and CtBP (C-terminus Binding Protein), thereby repressing target gene expression [13]. Upon Wnt stimulation, $\beta$-catenin is stabilized and translocated into the nucleus. Nuclear accumulation leads to interaction of $\beta$-catenin with Tcf/Lef which promotes specific gene expression [11].

In non-vertebrates transcriptional activation and repression is regulated solely by a single tcf gene, for example pangolin in Drosophila and POP-1 in C. elegans, whereas vertebrates have four, more specialized and partly redundant homologues (tcf1, tcf3, tcf4 and lef1) [11]. While Tcf1 and Tcf4 proteins play a role in both activation and repression, Lef1 primarily functions as an activator [14] and Tcf3 appears to function exclusively as a repressor [15]. In mice, tcf3 knockout experiments resulted in gastrulation defects that resemble ectopic Wnt expression such as duplication of the anterior-posterior (AP) axis. At later stages abundant neuroectodermal cells and defective neural patterning were observed [16]. In addition, Tcf3 seems to be a regulator of pluripotency. Embryonic stem (ES) cells show high levels of Tcf3 and only low Wnt pathway activity, thereby remaining in a balanced state between pluripotency and differentiation. When $t c f 3$ is knocked down or Wnt genes are overexpressed, the ES cells do not undergo efficient differentiation and the balance is tipped towards pluripotency [17, 18]. In zebrafish two tcf 3 genes have been reported, headless $h d l / t c f 3$ [19] and $t c f 3 b$ [20], which both act as repressors. At the shield stage (mid-gastrulation) $h d l$ is broadly expressed in the epiblast while tcf3b demonstrates only weak expression, at late gastrulation both genes are expressed in the rostral neuroectoderm and later on throughout the brain with a gap of expression in the mid-hindbrain boundary (MHB) [20]. In $h d l$ mutants the forebrain is lost while the expression of genes that characterize the MHB is expanded [19]. This phenotype can be rescued by $t c f 3 b$ overexpression, indicating redundant functions of $h d l$ and $t c f 3 b$ [20]. $t c f 3 b$ knockdown results in smaller heads but otherwise normal brain patterning, whereas interference with both tcf3 genes leads to strong caudalization [20].

One mechanism of Tcf3-mediated repression results from its interaction with Groucho/Tle proteins [13, 21, 22], which are transcriptional co-repressors that lack direct DNA-binding ability. Instead they bind to DNA-bound proteins and assist in repression (reviewed by Turki-Judeh and Courey [23]). In the case of Tcf3, Groucho/Tle tetramers bind to an $\mathrm{N}$-terminally located groucho interaction domain [24]. This interaction is disrupted by the Groucho/ Tle repressor Aes [21], which is a truncated form of
Groucho/Tle consisting only of the N-terminal Q- and GPdomains $[25,26]$.

Here we analyzed the function of tcf3 in medaka embryos using both gain- and loss-of-function experiments. For the knockdown we used pePNAs and morpholino oligonucleotides. Novel side chain modifications further improved the activity of the PNAs, resulting in knockdown efficiencies directly comparable to those of the morpholino oligonucleotides. Furthermore, we analyzed the effect of Groucho/Tle proteins on the repressive function of Tcf3 using a dominant negative approach and antisense oligonucleotides.

\section{Results}

\section{Isolation and expression of the medaka tcf3 gene}

We used a BLAST search to identify homologs of the tcf3 gene in the medaka genome, using the mouse Tcf3 protein sequence as a template. In this work, we identified several homologs of $t c f / l e f$ genes, however, only one single hit matched the $t c f 3$ gene (corresponding Ensembl transcript ENSORLT00000014810 located on chromosome 9 and encoding for a 600 amino acid protein). The presence of a single tcf3 gene in medaka was also reported previously [27]. In order to study the expression pattern of the medaka tcf3 gene, we isolated a cDNA fragment by RT-PCR and performed whole mount in situ hybridization (Fig. 1). During gastrulation, tcf3 is broadly expressed throughout the embryonic shield (40\% epiboly; Fig. 1a), followed by an anterior shift towards the prospective head region (Fig. 1c) and into the embryonic body (Fig. 1b,c) at late gastrula stage. At the neurula stage the expression becomes restricted to the head region (Fig. 1d,d') and appears in posterior regions of the embryo later during somitogenesis (Fig. 1e-h'). When the first subdivisions of the brain can be distinguished, tcf3 expression becomes segmented into the telencephalon, midbrain and hindbrain domains (Fig. 1e,e'). Similar to the expression of zebrafish $t c f 3$ [20], medaka $t c f 3$ is not expressed in the MHB (Fig. 1e-g). Furthermore, tcf 3 is also expressed in the eyes and the otic placodes (Fig. 1e). During somitogenesis, tcf3 expression further extends caudally (Fig. 1f-h'), where it is then found in the pectoral fins and the somites in late stages (Fig. $1 \mathrm{~h}, \mathrm{~h}$ '). Interestingly, the expression pattern of $t c f 3$ in medaka combines the expression domains of the zebrafish genes $h d l$ ( $t c f 3 a$ ) and $t c f 3 b[20]$.

\section{The medaka tcf3 gene is crucial for eye development}

In order to understand the function of the medaka $t c f 3$ gene during embryogenesis, we performed knockdown experiments using PNAs and morpholino oligonucleotides. We used a 16mer mixed pePNA (Fig. 2a) and a 25mer morpholino oligonucleotide sequence directed against the medaka $t c f 3$ mRNA directly upstream of the 


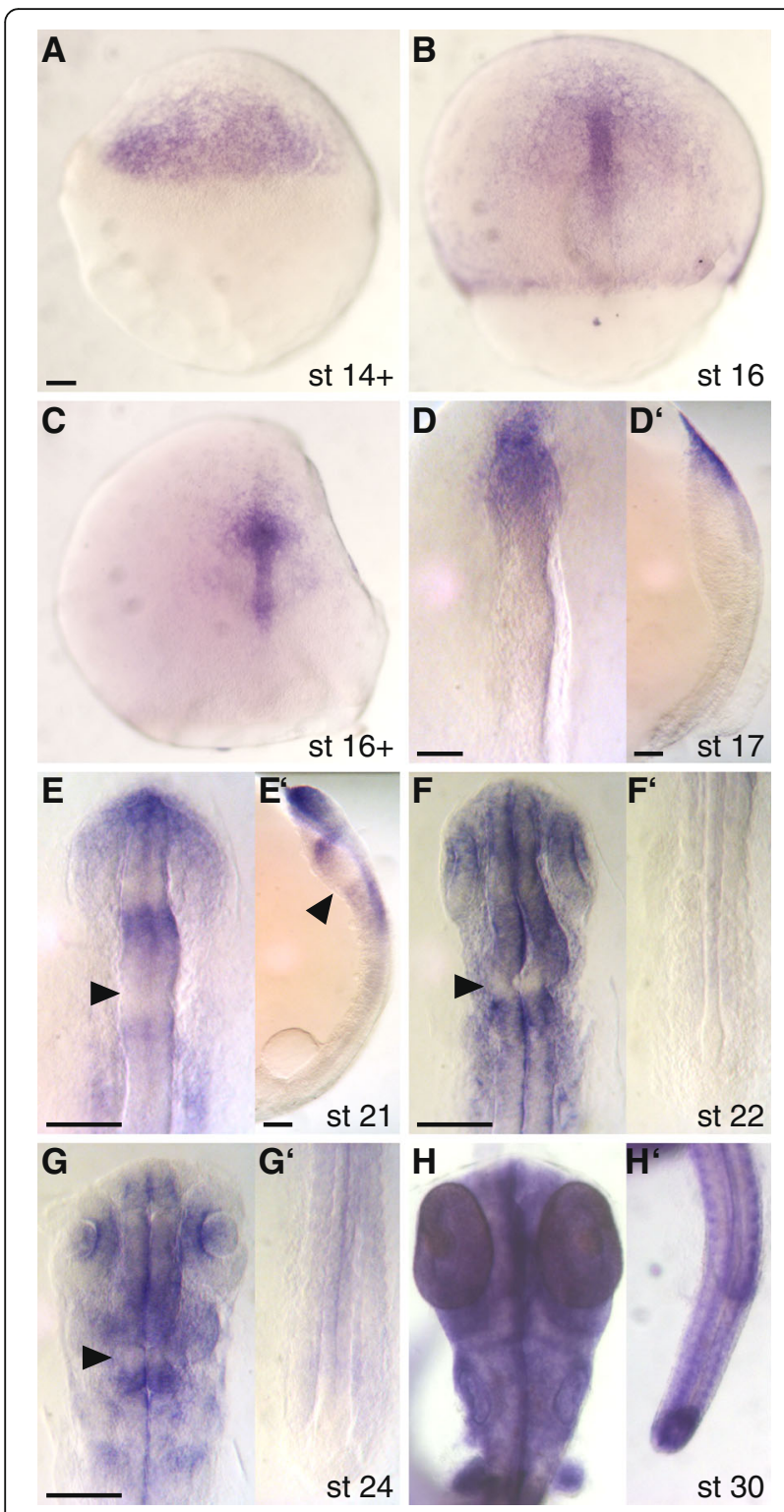

Fig. 1 tcf3 expression in medaka embryonic development. Whole mount in situ hybridization experiments were performed in wild type embryos at the indicated stages, using a digoxigenin-labelled RNA probe against tcf3. Embryos are shown in dorsal view, anterior at the top. (f-h) Flat mounts; (f'-h') tail view. During gastrulation (a-c) tcf3 is expressed throughout the epiblast and the embryonic body (c-d). In neurula the expression becomes restricted to the head (d,d') from where it spreads caudally and divides into a forebrain, midbrain, and hindbrain section (e;e'). A gap in expression at the mid-hindbrain boundary (f-h, arrowhead) is closed at later stages (h) In later stages, expression is observed throughout the entire body $\left(\mathbf{g}-\mathbf{h}^{\prime}\right)$. Scale bars $100 \mu \mathrm{m} ; \mathbf{a}, \mathbf{a}-\mathbf{c} ; \mathbf{f}, \mathbf{f}-\mathbf{f}^{\prime} ; \mathbf{g}, \mathbf{g}-\mathbf{h}^{\prime}$. Abbreviations: st, stage

translation start codon (Fig. 2b). Injection of PNAs and morpholino oligonucleotides resulted in the same phenotypes. The embryos progressed through gastrulation without apparent defects; however, eye development was later specifically impaired (Fig. 3b-d). For phenotype classification, we separated the embryos into three groups (weak, moderate and strong): weak embryos (Fig. 3b,f) exhibited one or two slightly smaller eyes than the control group, whereas in embryos with a moderate phenotype the size reduction was severe (Fig. 3c,g). Embryos without eyes were categorized as having a strong phenotype (Fig. 3d,h). Interestingly, in zebrafish the phenotypes for the two tcf3 genes differ considerably, however, in $h d l / t c f 3 b$ double morphants they add up to one combined phenotype representing the complete absence of Tcf3 proteins [20]. Medaka embryos with a strong phenotype correspond to this extreme case, whereas embryos of the weak and moderate groups demonstrate phenotypes in between those for $h d l[19]$ and $t c f 3 b$ [20] in zebrafish.

To further characterize the $t c f 3$ loss-of-function phenotype, we next examined the expression of pax2, pax6, gbx1 and wnt1 (Fig. 3i-p), which is known to be affected by Tcf3 activity [20]. In those embryos the expression domains of all three genes were shifted anteriorly (Fig. 3m-o) compared to the wild type control embryos (Fig. 3i-k). In $48 \%$ of the embryos $(n=61)$ the expression domain of the forebrain marker pax6 was smaller (Fig. 3m) and instead of two separate domains on either side of the prospective head (Fig. 3i), only a single cap-like structure was observed at the most anterior region of the prospective neural domain (Fig. 3m). However, we did not see a complete loss of anterior pax6 expression. The expression of pax2, a marker of the MHB, was shifted anteriorly in $56 \%$ of the morphants $(n=36)$, partially overlapping with the pax6 domain (Fig. 3n) which is in good agreement with the expression pattern found in zebrafish $h d l$ mutants [20]. In $37 \%$ of the embryos $(n=43)$ an altered expression of the hindbrain marker $g b x 1$ was observed, where again the entire expression domain was shifted anteriorly (Fig. 3o). In addition, the lateral edges of less severely caudalized embryos pointed anteriorly and in more severe phenotypes, the expression expanded into an arc-like shape, thereby overlapping with the expression domains of both pax6 and pax2. We also analysed the MHB marker wnt1 in tcf3 knockdown embryos and found expression in a more anterior position (Fig. 3b). Taken together, the expression of all marker genes clearly indicates the expected anterior shift for $t c f 3$ inactivation.

\section{Improvement of PNA specificity}

Qualification of the pigmented retinal structures of the eyes allowed a simple but reliable phenotype classification. Based on these results we could compare the knockdown efficiency of the different antisense molecules. We first used a mixed pePNA (Tcf3PNA), which contains two tri-methyllysine (TML) terminal groups. For each concentration the 


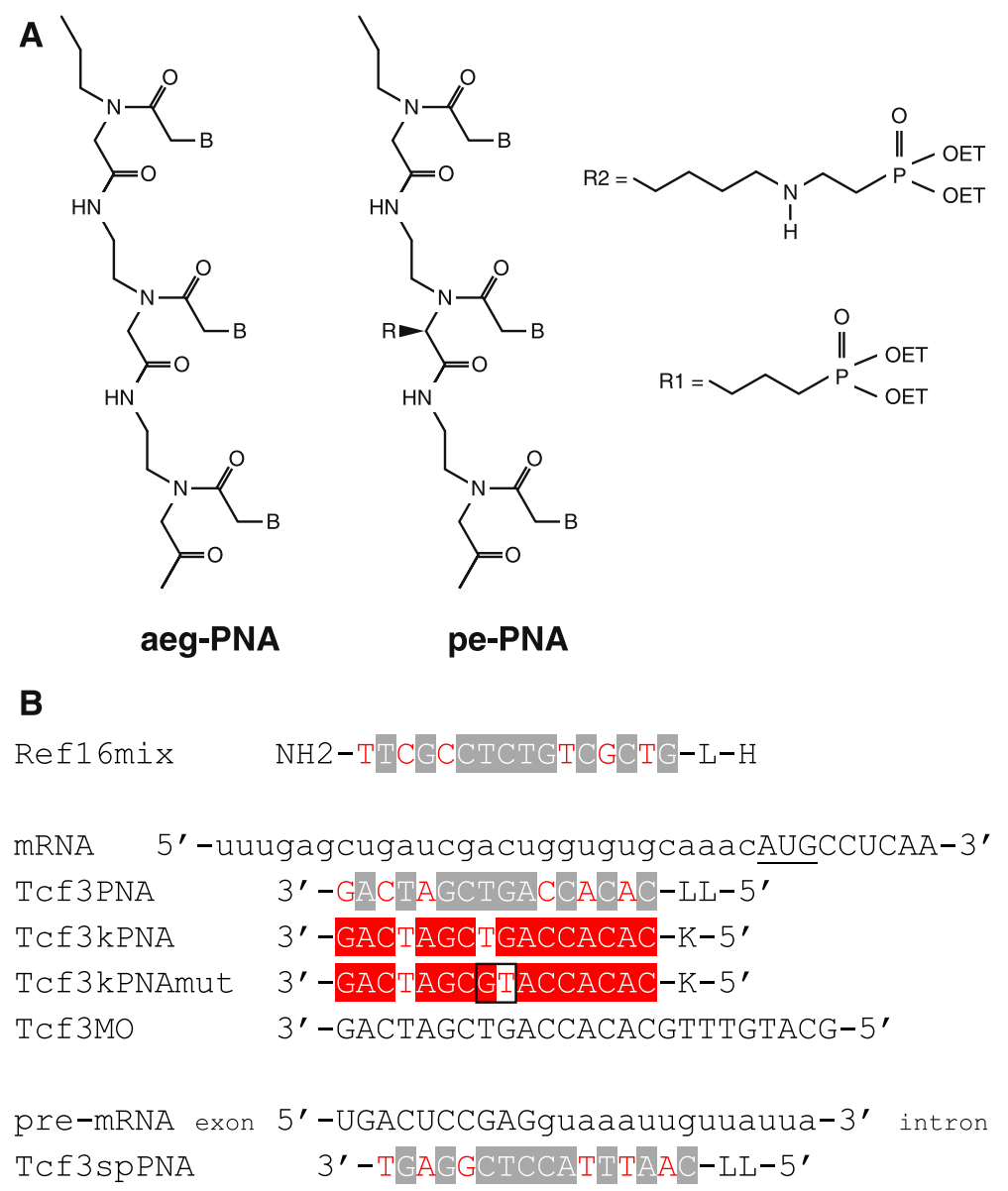

Fig. 2 Sequences of medaka tcf3 antisense molecules and their targets. a Schematic chemical structure of aegPNAs, pePNAs. Mixed PNAs contain C3- (R1) or lysine-phosphonic-ester- (R2) residues. b mRNA targets are shown in the 5'-3' orientation, capital letters indicate the coding region. The AUG start codon is underlined. Pre-mRNA represents the unspliced mRNA precursor of the medaka tcf3 gene with exon 1 sequences shown in capital letters (exon 1 includes the first 62 amino acids of Tcf3). Tcf3MO represents a morpholino oligonucleotide, all other sequences PNAs. aegPNA components are shown with grey overlay, pePNA components with C3-phosphonic-ester (R1) side chains are shown in red letters, those with lysine-phosphonic-ester (R2) side chains in red overlay. Boxed bases represent mismatches. All PNA and morpholino oligonucleotides are shown in $3^{\prime}-5^{\prime}$ orientation. $L$ represents a trimethyl-lysine and $\mathrm{K}$ a dimethyl-lysine

number of phenotypes of the different groups was determined and the total number of tcf3 knockdown phenotypes among the surviving embryos was calculated (Fig. 4a and Additional file 1). Between $100 \mu \mathrm{M}$ and $400 \mu \mathrm{M}$ of Tcf3PNA the total number of phenotypes increased from $22 \%$ to $51 \%$. At $600 \mu \mathrm{M}$ and $900 \mu \mathrm{M}$ the overall frequency of phenotypes (both $57 \%$ ) and also the number of dead embryos (6\% and $7 \%$, respectively) remained unchanged, which indicates a low toxicity. At $1200 \mu \mathrm{M}$ the percentage of tcf3specific phenotypes increased to $86 \%$, however, the mortality rate also increased to $38 \%$, indicating elevated toxicity of the PNA at higher concentrations. As expected, these increasing numbers of phenotypes also resulted in a continuous shift from preferentially weak phenotypes at low concentrations to a high proportion of strong phenotypes at high concentrations
(Additional file 1). The morpholino oligonucleotide showed phenotypes indistinguishable from the pePNAs, but differed in their efficiency. High rates of tcf3-specific phenotypes, $71 \%$ and $92 \%$, already appeared at low concentrations, $50 \mu \mathrm{M}$ and $100 \mu \mathrm{M}$, respectively. However, at $200 \mu \mathrm{M}$ the mortality rate jumped to $44 \%$, indicating toxic effects (Fig. $4 \mathrm{~b}$ and Additional file 1). At concentrations with high mortality rates, some embryos with unspecific phenotypes appeared which looked similar for both pePNAs and morpholino oligonucleotides. We previously described such unspecifically affected medaka embryos, which have smaller eyes but show severe defects over the entire brain [9]. Such unspecific phenotypes were not detected at non-toxic concentrations. Taken together, the morpholino oligonucleotide was more efficient than the pePNA at low concentrations, but also showed higher toxicity. 

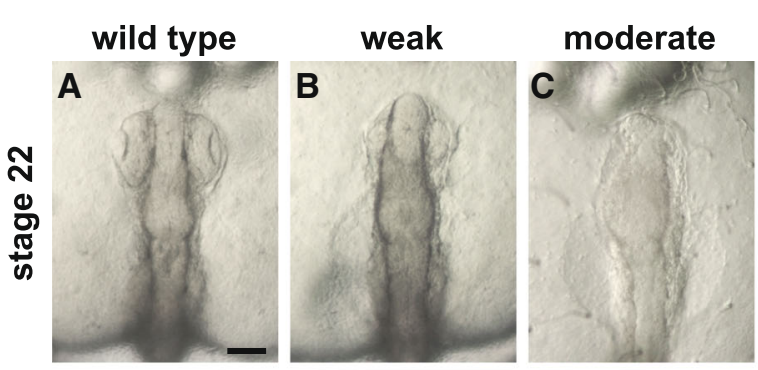

\section{strong}
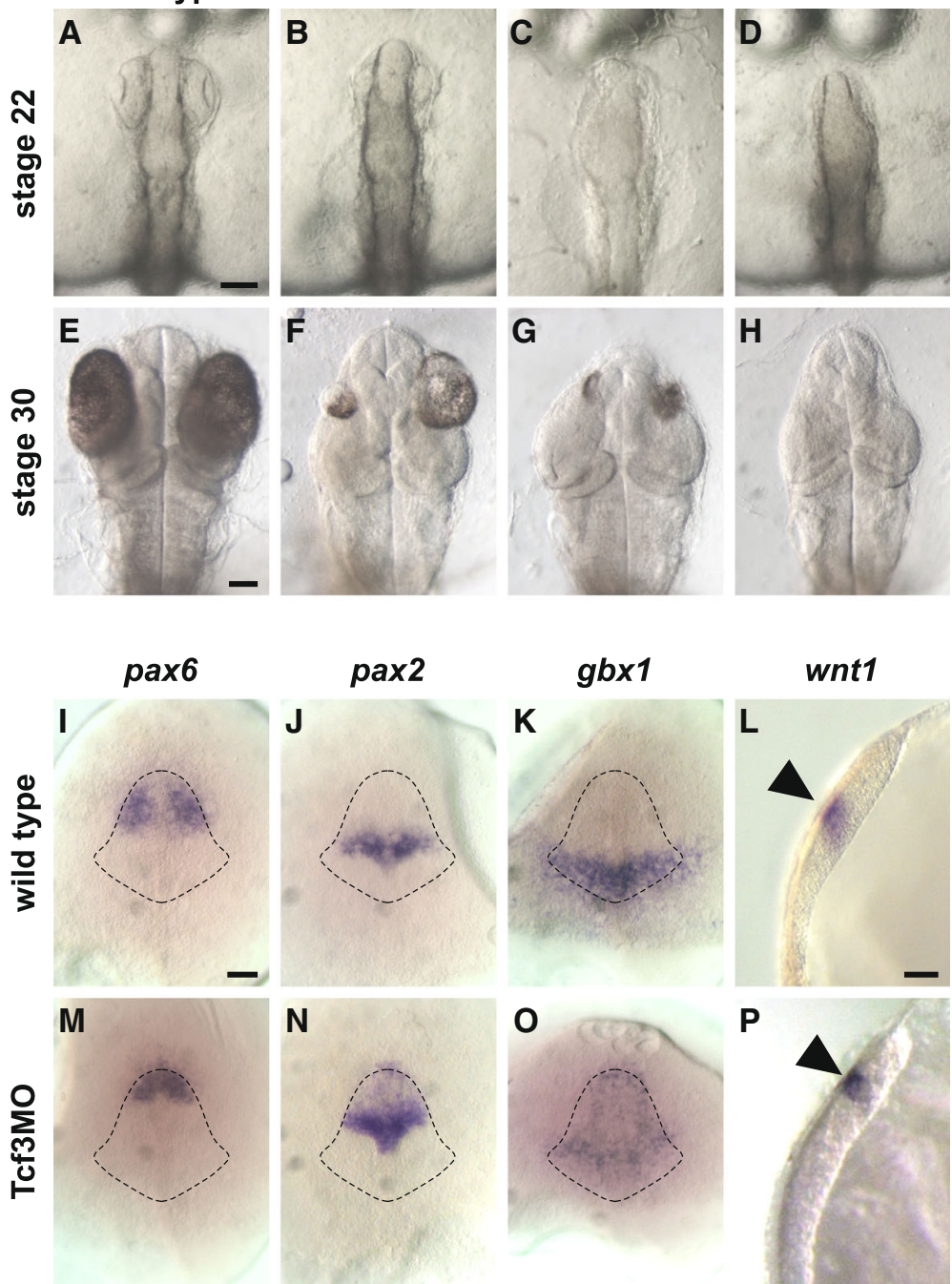

Fig. 3 Loss of function phenotypes for the tcf3 gene in medaka. Embryos at the 1-cell stage were co-injected with $300 \mu \mathrm{M}$ morpholino oligonucleotide (Tcf3MO) and $1 \mathrm{\mu g} / \mathrm{mL}$ FITC-dextran. The pairs $\mathbf{b}$ and $\mathbf{f}, \mathbf{c}$ and $\mathbf{g}$, $\mathbf{d}$ and $\mathbf{h}$ each show the same embryo at different stages (stage 22, a-d; stage 30, e-h). Control embryos $(\mathbf{a}, \mathbf{e})$ were co-injected with $1 \times$ Yamamoto's and FITC-dextran. Pictures of embryos with a fluorescent signal were taken at the indicated stages.

Classification was performed after the onset of eye pigmentation, according to the size of the eye: (b,f) weak, slightly reduced size; (c,g) moderate, severe size reduction; (d,h) strong, eye-less. Embryos at stage $16(\mathbf{i - k}, \mathbf{n}-\mathbf{o})$ are shown in dorsal view, anterior to the top; except for (l/p) which are at stage 17 and shown in lateral view. For genotypic analysis (i-p), whole mount in situ hybridization experiments were performed on MO injected embryos and un-injected wild type

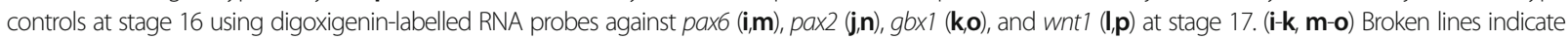
the outlines of the prospective neural domain estimated from the merged expression patterns formed by pax6, pax2 and gbx 1 in wild type embryos (i-k). An anterior shift of the expression domains was observed for all four genes. (l,p; arrowhead) wnt lexpression in the mid-hindbrain boundary. Scale bars 100 um; a, $\mathbf{a}-\mathbf{h} ; \mathbf{i}, \mathbf{i}-\mathbf{k}$ and $\mathbf{m}-\mathbf{o}$; I, I and $\mathbf{p}$. Abbreviations: MO, morpholino oligonucleotide

Thereafter, we tested the pePNAs' splice blocking efficiency. A sequence overlapping with both exon 1 and intron 1 was selected and also synthesized as a mixed PNA with a double TML end group (Tcf3spPNA; Fig. 2b). The result of this splice block is a truncated and therefore nonfunctional protein. Upon PNA injection the same phenotypes were observed as were found for Tcf3PNA, however, with lower efficiency (Fig. 4a and Additional file 1). The highest number of specifically affected embryos (49\%) was observed at a concentration of $900 \mu \mathrm{M}$. At $1200 \mu \mathrm{M}$ toxicity became dominant, similar to that seen for Tcf3PNA. We reasoned that a combination of both the translational blocking and the splice blocking PNAs might result in an improved knockdown efficiency. Indeed, coinjection of Tcf3PNA and Tcf3spPNA, each at a concentration of $200 \mu \mathrm{M}$, resulted in $50 \%$ of the embryos showing phenotypes (Fig. 4a and Additional file 1), which is higher than the percentage of affected embryos for both $400 \mu \mathrm{M}$ Tcf3PNA (39\%) and $400 \mu \mathrm{M}$ Tcf3spPNA (30\%). Therefore, the combined injection of both PNAs led to a synergistic 


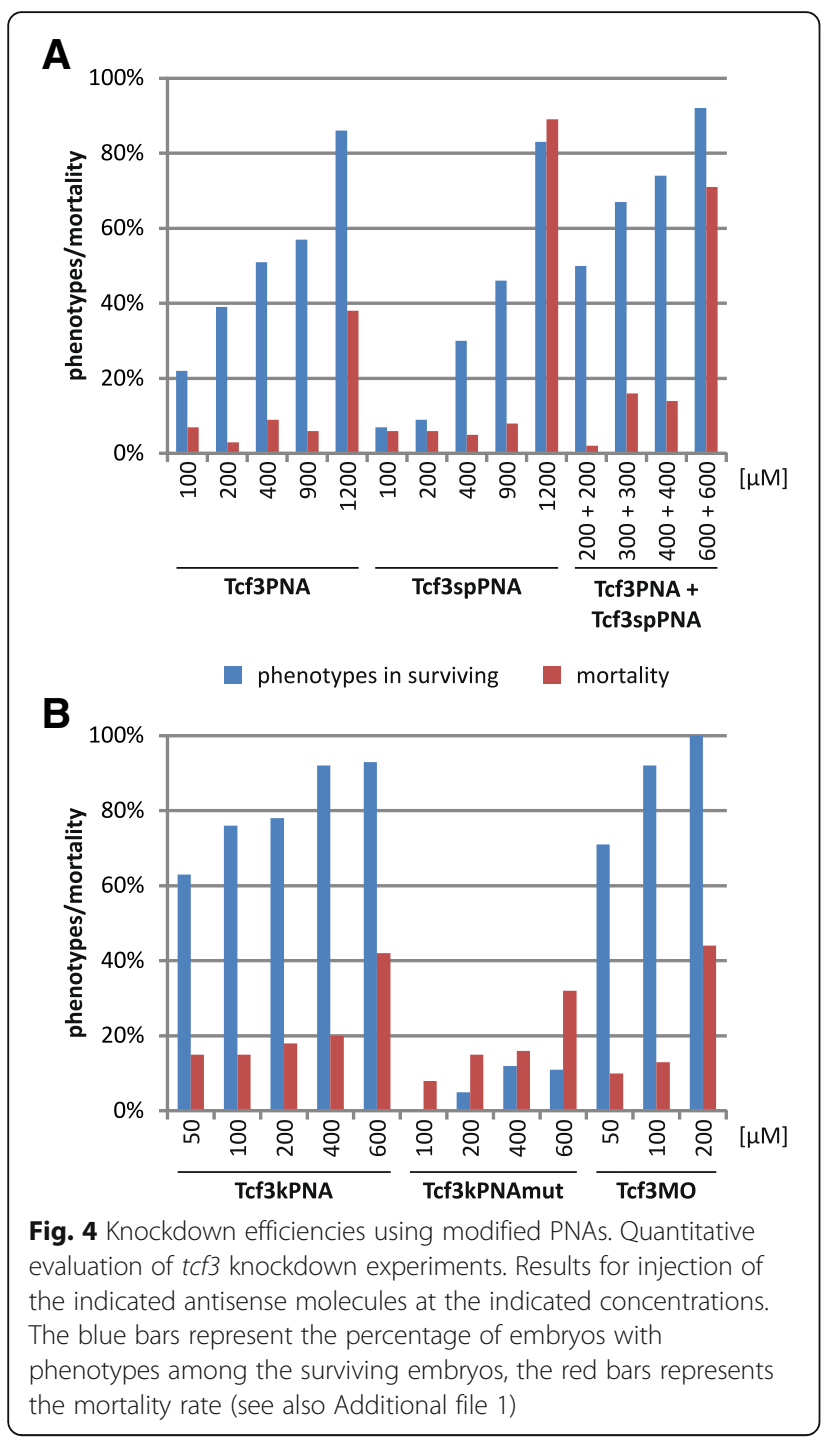

improvement, which was also observed at higher concentrations. At $900 \mu \mathrm{M}$ (450 $\mu \mathrm{M}$ each) $74 \%$ of the embryos showed tcf3-specific phenotypes without toxic side effects (those first appeared at $1200 \mu \mathrm{M}$ ).

We then tested lysine-phosphonic-ester (lpe) side chains as a novel backbone modification of the PNAs (Fig. 2a). A mixed 16mer PNA with the same target sequence as Tcf3PNA was synthesized (Tcf3kPNA; Fig. 2b). Injection of these new PNAs resulted in identical tcf 3 specific phenotypes, but in considerably higher numbers (Fig. 4b and Additional file 1). At $100 \mu \mathrm{M}$ the Tcf3kPNA produced $76 \%$ tcf3 specific phenotypes, compared to $22 \%$ for the Tcf3PNA. Similar improvements were also seen for other concentrations. A peak level of $92 \%$ phenotypes was observed at $400 \mu \mathrm{M}$ with the first toxic effects of these modified PNAs appearing at $600 \mu \mathrm{M}$. Therefore, the lpeside chain modification strongly improved the antisense efficiency of PNAs. To test specificity, a two nucleotide substitution was introduced (GT to TG at positions 8 and 9 of the PNA; Tcf3kPNAmut Fig. 2b). This substitution resulted in a dramatic loss of the $t c f 3$ knockdown phenotypes (Fig. 4b and Additional file 1). At $100 \mu \mathrm{M}$ none of the embryos showed tcf3-specific phenotypes and at $600 \mu \mathrm{M}$ only $11 \%$ did (compared to $93 \%$ for the nonmutated version). Therefore, a highly specific antisense function of the K-modified PNAs in an in vivo environment could be observed at low concentrations, directly comparable to the results for morpholino oligonucleotides. Taken together, the repeated appearance of the same phenotypes with various different antisense molecules clearly demonstrated specificity of the $t c f 3$ knockdown experiments.

\section{Tcf3 function in eye development is mediated by Groucho/Tle interaction}

In order to understand the effect of Tcf3 misexpression during embryogenesis, we injected a heat-inducible $t c f 3$ construct into the blastomere of one-cell stage wild-type embryos. The heat-inducible promoter allowed us a stagedependent gain-of-function analysis [28]. We first performed heat treatment at mid-gastrulation (stage 14/15) and analyzed the injected embryos after the onset of eye pigmentation at stage 30 . In this context, $31 \%(n=27)$ of the injected embryos showed reduced eye size and $8 \%$ lacked eyes; neither varying DNA concentration nor heatinduction time altered the eye phenotypes. Hence, based on our results gain-of-function and loss-of-function experiments for $t c f 3$ resulted in similar phenotypes.

We subsequently focused on the functional domains of Tcf3 which mediate transcriptional repression. More precisely, the Tcf3 protein contains two repression domains: a CtBP interaction domain at the C-terminus [29] and a Groucho/Tle interaction domain at the N-terminus [24] (Fig. 5a). We therefore constructed differently truncated tcf3 cDNAs that either lack the CtBP interaction domain (Tcf3 (1-434)), or both the CtBP and the Groucho/Tle interaction domain (Tcf3 (1-434) $\Delta$ Gro). Injection of Tcf3 (1-434) cDNA lacking the CtBP binding site, increased the frequency of embryos showing eye phenotypes to $26 \%$ (Fig. 5f) compared to $8 \%$ for the full-length Tcf3 construct. This indicates that CtBP interaction does not increase eye phenotypes, but instead reduces them. On the other hand, injection of the truncated $t c f 3$ cDNA lacking both the CtBP- and Groucho/Tle interaction domains (Tcf3 (1-434) $\Delta$ Gro), resulted in a significantly reduced frequency of embryos with strong eye phenotypes (5\%), which suggests that the Groucho/Tle interaction of Tcf3 is more important for eye development than that with $\mathrm{CtBP}$ is.

Additionally, we examined the expression of the retina-specific homeobox gene $r x 2$ in the injected embryos an early stage of eye development [30, 31]. In agreement with the eye phenotypes, three different 

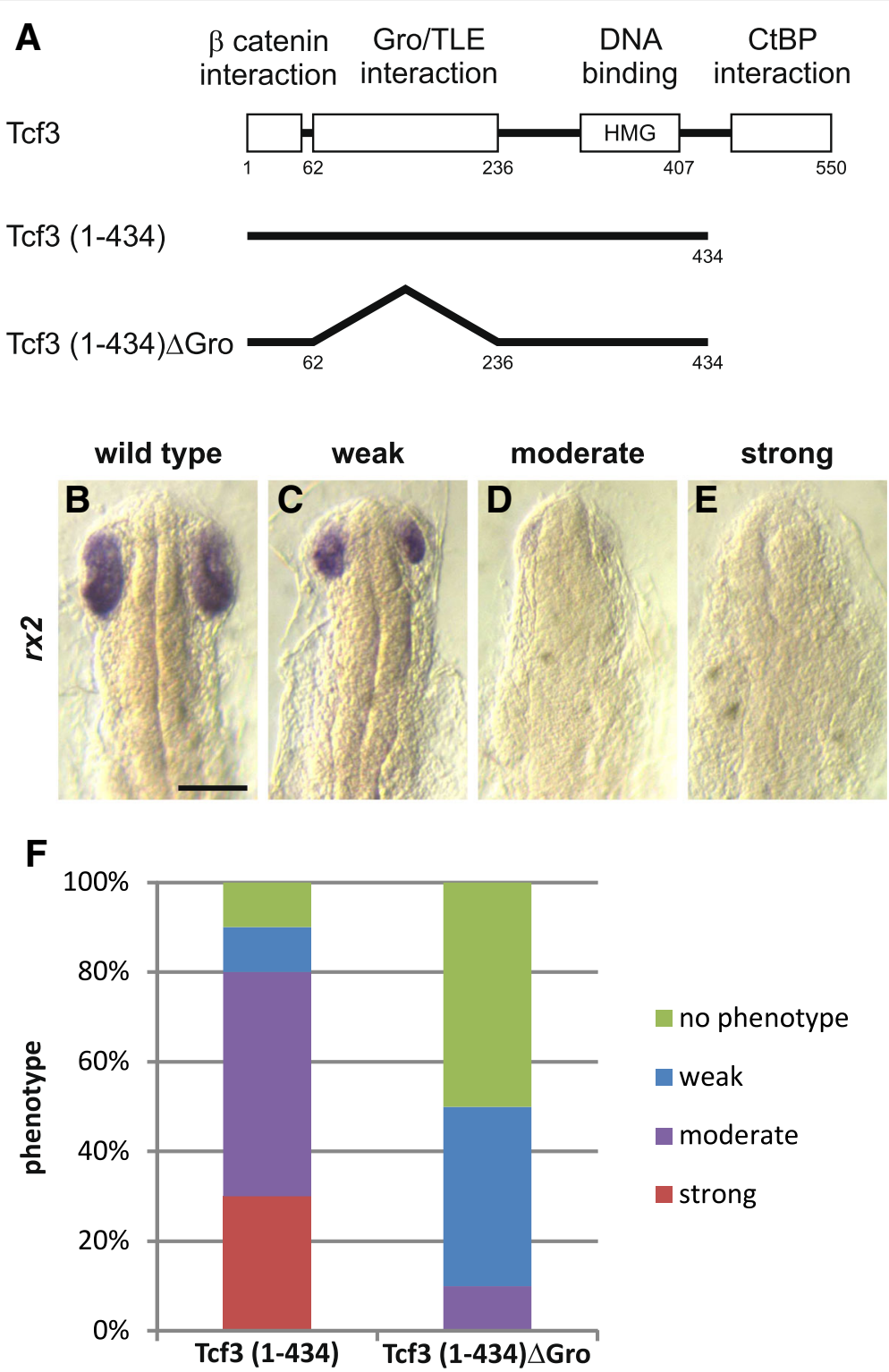

Fig. 5 tcf3 gain of function phenotypes. a Schematic representation of full length Tcf3, Tcf3 lacking the CtBP binding site (Tcf3[1-434]), and a C-terminally truncated Tcf3 lacking the Groucho/Tle interaction domain (Tcf3[1-434] $\Delta$ Gro). b-f Embryos at the 1-cell stage were injected with either heat-inducible truncated Tcf3 (Gfp:HSE:Tcf3(1-434); $40 \mathrm{ng} / \mu \mathrm{ll}$ ) or Tcf3 lacking the Groucho/Tle interaction domain (Gfp:HSE:Tcf3[1-434] $\Delta$ Gro; $40 \mathrm{ng} / \mu \mathrm{l})$. Heat induction was performed at stage 14-15. Gfp positive embryos were selected at stage 22 for subsequent whole mount in situ hybridization experiments using digoxigenin-labelled RNA probes against $r \times 2(\mathbf{c}-\mathbf{f})$. $\mathbf{b}$-e Flat mounts are shown in dorsal view, anterior at the top. Phenotypes were categorized according to their eye size and the expression intensity compared to the control: (c) weak, small eyes, normal expression intensity; (d) small eyes, weak expression; (e), missing eyes and expression. b Heat treated wild type control. f Quantitative results of the injections using $1 \times 2$ in situ hybridization. Scale bar $100 \mu \mathrm{m}$

expression patterns of $r x 2$ were observed in $t c f 3$-injected embryos at stage 21 (Fig. 5b-e). We compared the expression pattern of $r x 2$ in embryos injected either with Tcf3 (1-434) lacking the CtBP binding site or Tcf3 (1-434) $\Delta$ Gro lacking both the CtBP and the Groucho/ Tle interaction domains. The expression of $r \times 2$ was less reduced in embryos injected with the Tcf3 construct that lacks the Groucho/Tle interaction domain but still retains the DNA binding- and the $B$-catenin interaction domains (Fig. 5 f and Additional file 2). In particular, misexpressing this truncated version significantly reduced the appearance of eye phenotypes up to $50 \%$ in the injected embryos. Our data therefore suggest that Groucho/Tle co-repressor proteins make a crucial contribution to eye development. Moreover, in addition to eye phenotypes, $21 \% \quad(n=66)$ of $\mathrm{Tcf} 3(1-434) \Delta$ Gro injected embryos exhibited ectopic otic vesicles (Additional file 2B,C). We observed ectopic otic vesicles in the trunk and tail regions 
of the developing embryos, hence, posterior to the endogenous vesicles (Additional file 2C). Taken together, our results suggest that Tcf3 function in eye development is modulated by the Groucho/Tle corepressor interaction.

\section{Two Groucho/Tle genes, tle1 and tle2b, have redundant functions in eye development}

Groucho/Tle co-repressor proteins have been implicated in eye development [32, 33]. Particularly Tle1 and Aes were shown to interact with the transcription factors Six 3 and Six6 during early eye development [33]. However, several groucho/tle genes are broadly expressed in various developing tissues [34]. Thus, in order to interfere with the function of all Groucho/Tle proteins within one experiment we selected a dominant negative strategy and used a truncated form of Tle4 that contains only the $\mathrm{Q}$ domain. The Tle-Q domain is sufficient for interaction with Lef/Tcf proteins and forms a tetrameter upon binding to Lef1 [22]. The Aes protein, which is a truncated member of the Groucho/Tle family, contains only the N-terminal Q and GP domains (Fig. 6a). It has been used repeatedly as a dominant negative form for Groucho/Tle proteins [24, 35] forming intra- as well as intermolecular interactions with other Groucho/Tle proteins via its Q-domain. The resulting tetramers therefore will partially lack the $\mathrm{C}$-terminal domains, which are not present in the Aes protein. Most important at the C-terminus are the domains of WD40 repeats which represent a major protein-protein interaction domain. Inefficient complex formation is therefore the consequence of Aes expression. However, a major criticism of this strategy is that important interactions also originate from the Q-domain. Compared to other Groucho/Tle family members, the Aes Q-domain shows a number of deviations in its amino acid sequence. It is therefore not clear how efficiently the Aes Q-domain can contribute to the interactions in a mixed protein complex. To address this issue, we tested the effect of Aes overexpression on an established Q-domain interaction. For this we selected the Tle-Six3 interaction, which has been shown to be critical for eye development [33], and performed mammalian two-hybrid experiments. Our results showed a stepwise reduction of both Tle1- and Tle4-Six3 complex formation upon increasing Aes concentrations, whereas a control interaction was not affected (Additional file 3). This indicates that Aes overexpression can interfere with both $\mathrm{N}$ - and $\mathrm{C}$-terminal interactions of Groucho/Tle proteins in the mixed complexes.

Based on these data we generated two heat-inducible transgenic lines for misexpression of Aes (HSE:Aes) and the Tle4-Q domain (HSE:Q). We then performed heat shock at mid gastrula stage and analysed the embryos over the following days (Fig. 6b-e). In agreement with previous studies we observed eye phenotypes [32, 33].
Interestingly in both transgenic lines a range of phenotypes appeared which might be explained by variations of the heat shock induction and the dependence of the dominant negative approach on the resulting expression levels. We used a phenotype classification scheme similar to the previous one. Embryos with a weak phenotype developed slightly smaller eyes which point towards the midline (Fig. 6c-c"), whereas embryos with a strong phenotype showed cyclopic eyes (Fig. 6e-e"). As a moderate phenotype we considered embryos between these extremes (Fig. 6d-d"). Here, additionally to the weak phenotype, the lenses were shifted anteriorly and mild cyclopia anterior of the forebrain was observed. In HSE:Aes embryos a total of $24 \%$ developed a phenotype $(n=97)$, equally distributed between weak and strong. The HSE:Q line showed a total of 34\% phenotypes among the induced embryos $(n=79)$, however, only $5 \%$ of the embryos were categorized as having a strong phenotype. Therefore, the interference of the Q-domain was less efficient compared to the aes-misexpressing line, but interestingly the phenotypes were identical. We then analysed the expression pattern of marker genes for retina ( $r x 2$ ), telencephalon (bf1) and midbrain (wnt1) development in the transgenic embryos at stage 21 (Fig. 6f-k). Supporting the eye phenotypes, the expression of $r x 2$ was present but restricted to eye size (Fig. 6i). In embryos with a strong phenotype, an additional faint stripe of $r x 2$ expression that connected the two optic vesicles was observed in the forebrain. In those embryos the expression of $b f 1$ was also impaired (Fig. 6j). Furthermore, the expression of wht1 indicated a slight reduction in the size of the midbrain (Fig. 6k).

In order to elucidate which of the groucho/tle genes are involved in eye development we specifically impaired the function of individual genes using antisense oligonucleotides. In medaka, six full length members of the groucho/tle family were described; most of them are expressed in largely overlapping domains indicating that they might have redundant functions [34]. We selected three groucho/tle paralogs (tle1, tle $2 b$ and tle3b) that are specifically expressed in the optic placode [33, 34]. Interestingly, we observed similar eye phenotypes for all three genes. According to our phenotype classification $10 \%-16 \%$ of the morphants showed eye defects (for more details see Additional file 4), in which the tle $2 b$-morphants showed the highest frequencies in strong- $(16 \%$, $n=237)$ and eye-less (7\%) phenotypes. We next tested combined inactivation and observed an additive effect in tle1-tle $2 b$ morphants $(27 \%, n=44)$ but not in triple morphants $(17 \%, n=58)$. The appearance of eye defects in tle morphants fully supported our transgenic HSE:aes and HSE:Q results. Nevertheless, we observed some slightly different phenotypes resulting from these two 
A

\begin{tabular}{|c|c|c|c|c|}
\hline Tle1-4 $\longdiv { Q }$ & GP & $\mathrm{CcN}$ & SP & WD 40 \\
\hline
\end{tabular}

$\mathbf{Q} \mathbf{Q}$

Aes \begin{tabular}{|l|l|}
\hline $\mathbf{Q}$ & GP \\
\hline
\end{tabular}
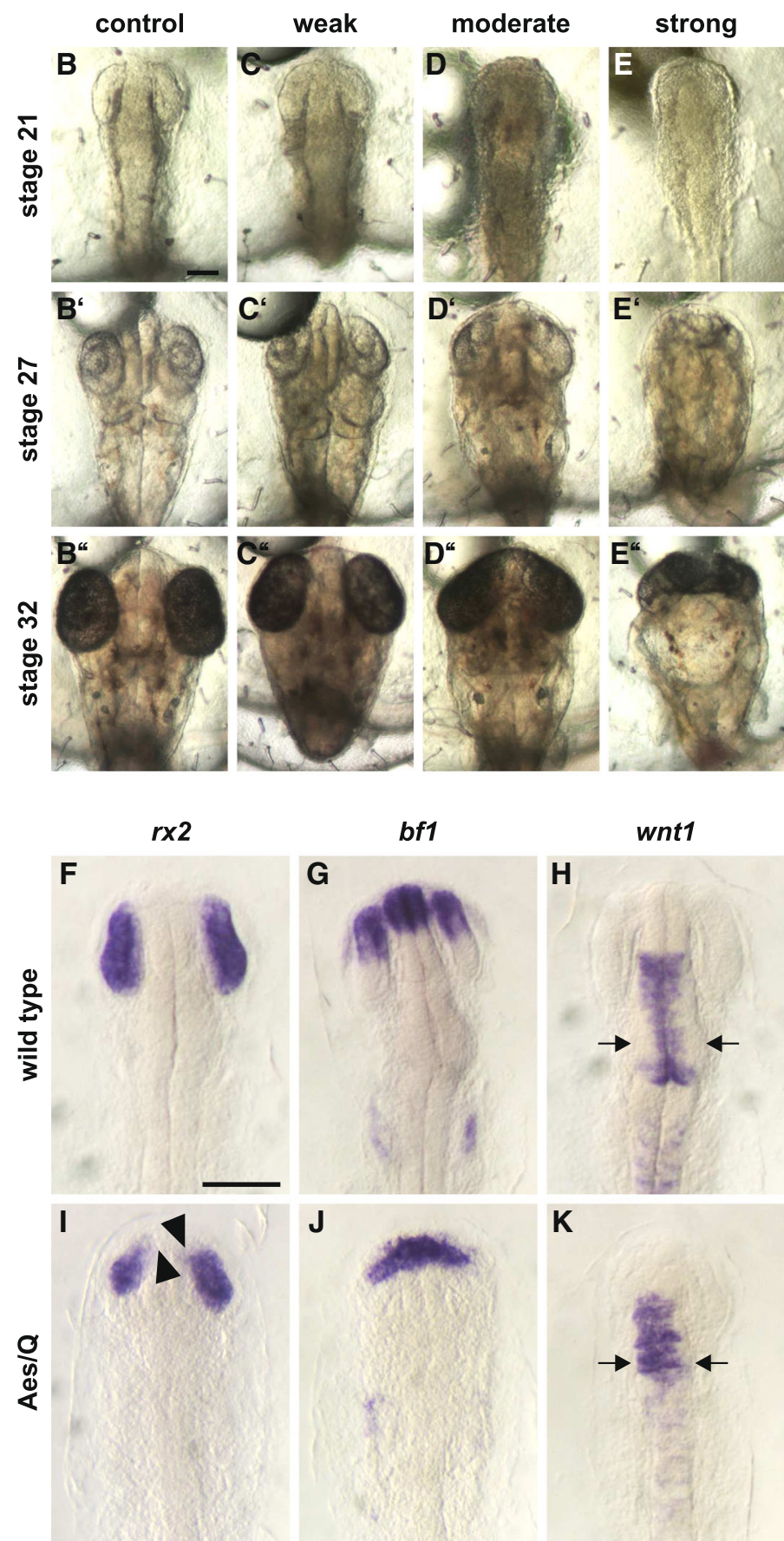

bf1

wnt1
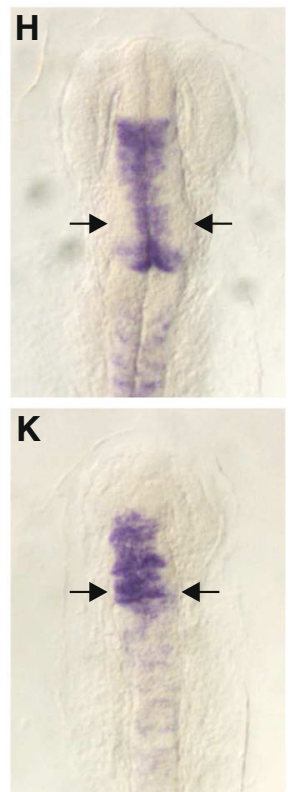

Fig. 6 (See legend on next page.) 
(See figure on previous page.)

Fig. 6 Aes-mediated Groucho/Tle loss of function phenotypes. a Schematized presentation of full length Tle protein, the Q-domain, and Aes. For transgenic lines, embryos at the 1-cell stage were injected with $20 \mathrm{ng} / \mu \mathrm{l}$ of DNA (Gfp:HSE:Aes and Gfp:HSE:Q). The F1 generation was heat-induced (10 min, $\left.43.5^{\circ} \mathrm{C}\right)$ at stage 15/16. b-k Embryos are shown in dorsal view, anterior to the top. f-k Flat mounts. c-d Phenotypes of the Aes/Q-mediated Groucho/Tle loss of function were categorized according to their eye phenotype into: (c-c') weak, smaller eyes tilted towards the midline; $\left(\mathbf{d}-\mathbf{d}^{\prime \prime}\right)$ moderate, beginning cyclopia anterior; strong (e-e'), cyclopic eye. For whole mount in situ hybridization experiments (f-k), using the indicated digoxigenin-labelled RNA probes, fixation was performed at stage 21; heat treatment occurred as described before. $\mathbf{i} r \times 2$ expression indicates a reduction in eye size, the eyes are tilted at the anterior end towards the midline (arrowheads); (j) Bf1 expression is reduced; (k) Wnt1 expression indicates a reduction in midbrain size. Instead of a defined expression close to the midline (h, arrows), Aes misexpression resulted in an indistinct expression pattern throughout the midbrain (K, arrows). (b-b", $\mathbf{f - h}$ ) control embryos were heat-treated wild type embryos. Scale bars $100 \mu$ m; b, b-e"; f, f-k

approaches, in particular the cyclopic eye connection in the tle 1 morphants appeared in the middle of the eyes (see Additional file 4C), whereas these connections were preferentially observed anterior to the forebrain in the transgenic HSE:Aes and HSE:Q embryos (Fig. 6d").

\section{Discussion}

\section{Knockdown of the tcf 3 gene in medaka resembles the} zebrafish $\mathrm{hdl} / \mathrm{tcf} 3 \mathrm{~b}$ double morphants

The expression and function of tcf3 in medaka is poorly understood. In contrast to zebrafish, medaka has only a single tcf3 gene (this study and [27]), however the expression pattern closely resembled that of the zebrafish $h d l$ and $t c f 3 b$ genes (Fig. $1 ;[19,20])$. It was therefore not surprising that the phenotype of medaka tcf3 morphants resembled the combined inactivation of both zebrafish tcf3 paralogs (e.g. complete lack of eyes and anterior brain defects). Knockdown of medaka tcf3 resulted either in weakly or moderately affected embryos with reduced eye size, covering the range of phenotypes that have been seen for inactivation of single $t c f 3$ genes in zebrafish, or in strongly affected eye-less embryos corresponding to the complete absence of Tcf3 proteins in zebrafish $h d l / t c f 3 b$ double morphants (Fig. 3). The analysis of marker gene expression in the $t c f 3$ morphants verifies the strong effect on the Tcf3/Wnt gradient along the AP axis [36, 37], all marker genes tested in this study showed an anterior shift centering on the position of the prechordal plate. These anterior defects are also in good agreement with the AP defects seen in Wnt1 overexpression experiments [38]. Dorsky and colleagues analyzed the expression of these genes in zebrafish upon tcf3 inactivation. $H d l$ mutants showed a decrease in pax6 expression in the presumptive head region and pax2.1 expression was expanded rostrally, whereas the $g b x 1$ domain remained unchanged. However, if both $h d l$ and $t c f 3 b$ were inactivated, $g b x 1$ also expanded anteriorly, thereby including the expression domain of pax2.1, and the anterior expression of pax6 was completely lost [20]. This stepwise reduction of Tcf3 activity in zebrafish resulted in a progressive anterior shift of the marker genes centring on the position of the prechordal plate [36]. Taken together, a single tcf3 gene in medaka combines the function of the two tcf3 paralogous genes in zebrafish.

\section{Novel side chains provide higher antisense efficiency for PNAs}

In this study, we also applied PNAs for tcf3 knockdown experiments in medaka embryos. We used phosphonic ester modified mixed PNAs, which previously were shown to be suitable for loss-of-function experiments in medaka [9]. Here again we could demonstrate a specific knockdown of gene function. Initially we used pePNAs with two TML end groups and obtained almost $60 \%$ specific phenotypes (400 to $900 \mu \mathrm{M})$ (Fig. 4a and Additional file 1). At higher concentrations this percentage further increased, however, also unspecific toxicity was observed. The pePNA molecules were also effective in a spliceblocking approach. Although the efficiency was lower compared to the translational blocking experiments, the same $t c f 3$ specific phenotypes appeared (Fig. 4a and Additional file 1). Furthermore, a combined injection of both PNAs synergistically improved the overall efficiency of the antisense molecules (74\% specific phenotypes).

Morpholino oligonucleotides resulted in higher efficiency when directly compared with pePNAs (e.g. 92\% and $22 \%$ phenotypes at $100 \mu \mathrm{M}$, respectively). However, also toxic effects started at considerably lower concentrations (200 $\mu \mathrm{M}$ and $1200 \mu \mathrm{M}$, respectively). Otherwise, the obtained phenotypes were identical for the two antisense molecules (Fig. 4b and Additional file 1). Interestingly, further modification of the PNA backbone with lpe-side chains (Fig. 2a) considerably improved the PNA efficiency (Fig. 4b and Additional file 1). At concentrations of $50 \mu \mathrm{M}$ to $400 \mu \mathrm{M}, 63 \%$ to $92 \%$ of the embryos showed $t c f 3$ specific phenotypes (with toxicity first appearing at $600 \mu \mathrm{M})$. This efficiency is in the same range as that for the morpholino oligonucleotides. An exchange of 2 nucleotides in this sequence (GT to TG at positions 8 and 9) resulted in a dramatic drop in phenotypes ( $8 \%$ to $16 \%$ phenotypes at $100 \mu \mathrm{M}$ to $400 \mu \mathrm{M}$ ), thus demonstrating the high specificity of this $16 \mathrm{mer}$ PNA. Therefore, novel side chain modifications efficiently improved the in vivo antisense function of PNAs. 


\section{Groucho/Tle corepressor proteins are critical for Tcf3 function in eye development}

The Tcf3 protein has two major co-repressor interaction domains; an amino-terminally located domain that interacts with Groucho/Tle proteins [24] and a carboxyterminal domain that interacts with the CtBP protein [29]. Our results indicate that the CtBP interacting domain in Tcf3 is dispensable for early embryonic eye development; indicating that the interaction with Groucho/Tle proteins is more crucial. In particular, Tcf3 variants lacking the Groucho/Tle interaction domain showed considerably reduced numbers of eye phenotypes in our gain-of-function experiments. These results further support the idea that the Groucho/Tle co-repressors are required for eye development [33, 39].

To interfere with the function of all Groucho/Tle proteins we used three strategies. Firstly, we misexpressed the aes gene. Aes (Fig. 6) is a naturally occurring truncated family member of full-length Groucho/Tle proteins that consists of only the Q and the GP domains $[25,26,40]$. The $\mathrm{Q}$ domain allows Aes to oligomerize with full-length Groucho/Tle proteins, thereby reducing the number of C-terminal interaction domains $[21,33,41-45]$. The GP domain, however, is functionally different from full-length Groucho/Tle proteins since it does not interact with HDAC corepressors [13, 39, 46, 47]. It was therefore suggested that through this interaction Aes turns Groucho/Tle oligomers into non-functional complexes, which therefore have a reduced number of C-terminal WD-40 interaction domains and a reduced efficiency to cooperate with HDAC co-repressors [21, 41, 42, 44, 48, 49]. Supportive of this hypothesis, our mammalian two-hybrid experiments using the Six 3 transcription factor, indicated that additionally N-terminal interactions with the Q-domain are blocked by Aes misexpression. Indeed, ectopic expression of Aes in medaka resulted in considerably smaller eyes and otic vesicles and reduced expression of optic and otic marker genes, suggesting an efficient block of Groucho/ Tle activity $[32,33]$. In addition to the Groucho/Tle blocking functions, Aes is also known to exhibit specific functions on its own in certain cellular contexts $[46,50,51]$. The observed phenotypes could therefore be a mixture between groucho/tle loss-of-function and aes gain-offunction effects. Secondly, we misexpressed the Tle4 Q-domain and could phenocopy the Aes misexpression embryos, however, at a lower efficiency. The reduction of the $b f 1$ and $w n t 1$ expression field in the forebrain and midbrain in those embryos also confirmed the observed phenotypes and is in agreement with a previous study in Xenopus [52]. Finally, we analysed the function of groucho/tle genes by injection of morpholino oligonucleotides. Previously, we have shown that three paralogs of medaka groucho/tle genes (tle1, tle $2 b$ and tle $3 b$ ) are expressed during early eye development [34]. Interestingly, knockdown of each of these genes, individually or in combination, resulted in specific impairment of eye development which suggests redundancy. These phenotypes closely resembled those of the dominant negative experiments and thus fully support this approach.

In addition to eye phenotypes, we also observed ectopic otic vesicles in the $\operatorname{Tcf} 3[1-434] \Delta$ Gro- injected embryos. The appearance of ectopic otic vesicles might be caused by a dominant negative effect of the overexpressed truncated Tcf3, thus blocking the repressing function of full-length Tcf3. Such an anti-repressive effect would be equivalent to ectopic Wnt activation. Indeed, we have previously shown that induction of Wnt signaling leads to the formation of ectopic otic vesicles [38, 53].

\section{Conclusion}

Taken together, our results suggest that a single tcf 3 gene in medaka is crucial for eye development and its function is modulated by interaction with Groucho/Tle co-repressor proteins. Gain-of-function analyses of Aes and the Q-domain, as dominant negative effectors, together with the loss-of-function analyses of individual tle genes confirm this interpretation. Moreover, our results indicate that three tle genes (tle1, tle $2 b$ and tle $3 b$ ) might have redundant functions during eye development.

\section{Methods}

\section{PNA and morpholino oligonucleotide synthesis}

Lysine-phosphonic-ester Ugi-PNA monomers were synthesized according to the following process. First, an $\mathrm{N}-\mathrm{Cbz}$ or $\mathrm{N}$-Boc protected phosphono glycinate was reacted with the appropriate aldehyde in a HornerWadsworth-Emmons [54-56] followed by enantioselective hydrogenation using Burk's catalyst [57-59] to give the $\alpha$-substituted protected glycinate. Deprotection of the $\alpha$-amino function and subsequent reductive amination with N-Boc-amino acetaldehyde gave the Ugi-PNAmonomer backbone. The latter was coupled to the appropriate protected nucleobase acetic acid component, employing HATU as coupling reagent. Finally, deprotection of the carboxylic function yielded the Ugi PNA monomer. Synthesis of the C3-phosphonic-ester-UgiPNA monomer building blocks was performed as described previously [9]. The PNAs were dissolved to $1 \mathrm{mM}$ in nuclease free water by repeated shaking and vortexting. Finally they were gently sonicated for $2 \mathrm{~min}$ with repeated pulses. Subsequently the PNAs were kept at $-80{ }^{\circ} \mathrm{C}$. Phosphorodiamidate morpholino oligonucleotides (abbreviated here as morpholinos) were manufactured by Gene Tools (see Fig. 3). Morpholino sequences for the tle genes in $5^{\prime}-3^{\prime}$ direction: tle1, CGCGTCTTGTCCTGAAACCCCGCTA; tle $2 b$, GGCA 
GTGCGTCCTCGTGGCTCTTTC; tle3b, CGGCCTT GTGGATACATGTCTCGTC. For injection into medaka embryos, morpholinos were dissolved in $1 \times$ Yamamoto's Ringer solution at the indicated concentration and injected into the blastomere at the onecell stage.

\section{DNA constructs}

All DNA constructs for microinjection were under the control of a bi-directional heat-inducible promoter [28] and contained either the mouse Aes/Grg5 cDNA, the mouse Tle4 Q domain (AA 1-135), or various deletion fragments of Tcf3 cDNA (see Fig. 5 for details). For cell culture experiments pMC [60] and pKC (polylinker modification of pKW) [61] were used (both vectors contain cytomegalovirus promoters). pLucF24ZF; luciferase reporter under the control of a Fos minimal promoter and ZFHD binding sites. pMChSix3(85-203)mZFb6; the human Six3 fragment (positions of amino acids in brackets) fused to a ZFHD DNA binding domain [62] and the "base" leucine-zipper [63]. pMCTle1VP16 and pMCTle4VP16; human Tle1 and mouse Tle4 were fused to the transcriptional activation domain of the herpes simplex virus protein VP16, respectively. pMCacidVP16 contains the "acid" leucine-zipper fused to VP16, pKCAes the mouse Aes/Grg5 cDNA.

\section{Microinjection into medaka embryos}

Embryos of the medaka Cab strain were used for all experiments. Adult fish were maintained at $26{ }^{\circ} \mathrm{C}$ with an artificial $14 \mathrm{~h}$ light and $10 \mathrm{~h}$ dark cycle. Stages were determined according to the morphological markers described in Iwamatsu [64]. DNA constructs were coinjected with I-SceI meganuclease enzyme $(0.5 \mathrm{U} / \mu \mathrm{l})$ at the one-cell stage. For transient studies, different versions of truncated tcf3 cDNAs (HSE:tcf3 constructs) were injected at a concentration of $40 \mathrm{ng} / \mu \mathrm{l}$. For the generation of a heat-inducible aes (HSE:aes) and Qdomain (HSE:Q) transgenic lines, DNA constructs were injected at $10 \mathrm{ng} / \mu \mathrm{l}$ concentration together with I-SceI meganuclease enzyme. The embryos were incubated at normal conditions ( $1 \times$ ERM buffer at $\left.27{ }^{\circ} \mathrm{C}\right)$. Heat treatment was performed at stage 14 (except if otherwise indicated) for $10 \mathrm{~min}$ at $43.5^{\circ} \mathrm{C}$. For co-injection experiments FITC-dextran (FD10S, Sigma-Aldrich) was used.

\section{Whole-mount in situ hybridization}

Whole-mount in situ hybridization using DIG-labelled RNA probes was performed as described previously [34]. Probes against tcf3 (Ensembl: ENSORLT00000014810) and $g b x 1$ (Ensembl: ENSORLT00000021915) were PCR amplified from medaka embryonic cDNA using specific primers for tcf3 (5'-CGGATCCATGGCTCAACTGAAC GGAGGC-3' and 5'-CGAAGACGGCTGGACATGGAT
GCATTCA-3') and $g b x 1$ (5'-TTAGAAAATACAGCCAC AA-3' and 5'-TCACTGTAAAAAGTACCTG-3'). The expression patterns of pax6 [65], pax2 [66], wht1 [67], $r x 2$ [30] and $b f 1$ [68] were described previously.

\section{Cell culture and mammalian two-hybrid assay}

HeLa cells $(300,194$, Cell Line Services) were transfected in polyethylenimine $(2.5 \mu \mathrm{g} / \mathrm{ml})$ coated 96-well plates [69] using TurboFect transfection reagent according to the instructions of the manufacturer (Thermo Scientific). For luciferase activity detection, additional transfection of $2 \mathrm{ng}$ Gaussia luciferase expression vector pMCGlucS served as an internal reference. Luciferase activities were measured $24 \mathrm{~h}$ after transfection. For the mammalian two-hybrid assay, cells were co-transfected with $70 \mathrm{ng}$ of firefly luciferase reporter construct pLucF24ZF, $20 \mathrm{ng}$ of Six3 expressing plasmid pMChSix3(85-203)mZFbase (six-domain of human Six3), 0-6 ng of Aes expressing plasmid pKCAes, and $20 \mathrm{ng}$ of either pMCTle1VP16, Tle4 pMCTle4VP16 or the control pMCacidVP16.

\section{Additional file}

Additional file 1: Overview of the PNA and MO induced loss of function phenotypes. Embryos at the 1-cell stage were injected with PNAs or MOs at the indicated concentrations. Phenotypes were categorized according to the size of the eyes into: weak, slightly smaller eyes; moderate, smaller eyes; strong, eye-less. Abbreviations: PNA, peptide nucleic acid; MO, morpholino oligonucleotides. (PDF $10028 \mathrm{~kb}$ )

Additional file 2: Gro/Tle dependence of 1 tcf3 in gain-of-function experiments. Embryos at the 1-2 cell stage were co-injected with $40 \mathrm{ng} / \mu \mathrm{l}$ of the indicated gfp:HSE:Tcf3 constructs. Heat treatment $\left(10 \mathrm{~min}, 43.5^{\circ} \mathrm{C}\right.$ ) was applied at stage 14 . (A) Statistical overview of the phenotype distribution. Whole mount in situ hybridization experiments for rx2 were performed on embryos at stage 21. (B) dorsal view of a stage 31 embryo with anterior at the top, (C) lateral view with anterior at the left. Arrowheads indicate ectopic otic vesicles, the arrow points to the endogenous otic vesicle. Scale bar $100 \mu \mathrm{m} ;$ B and C. (PDF 10028 kb)

Additional file 3: Mammalian two-hybrid analysis of Aes-mediated Gro/ Tle repression. $20 \mathrm{ng}$ of the expression constructs (pMCamVP16, pMCTle4VP16 or pMCTle1VP16) were co-transfected with 70 ng of firefly luciferase reporter construct pLucF24ZF, 20 ng of the expression construct for the six-domain of human Six3 (pMChSix3(85-203)mZFb6) and full length Aes (pKCAes at the indicated concentrations) into HeLa cells. The interaction of the artificial leucine zipper domain "acid" and "base" [63] was used as a control. All three interactions were set 100\% in the absence of Aes (acid/base corresponds to a more than 100 fold activation of the luc reporter construct compared to a control without bait, similar induction rates were seen for Tle4/Six3 and Tle1/Six3). Addition of the Aes expression construct at the indicated concentrations did not affect the acid/base interaction (blue bars), but strongly reduced the Tle4/Six3- (red bars) and the Tle1/Six3 interaction (green bars). (PDF $10028 \mathrm{~kb}$ )

Additional file 4: $\mathrm{MO}$-induced gro/tle loss of function phenotypes. Embryos at the 1-cell stage were co-injected with morpholino oligonucleotides and $1 \mu \mathrm{g} / \mathrm{ml}$ FITC-dextran. Injections were performed using either a single morpholino directed against tle $(\mathrm{C})$, tle2b $(\mathrm{D}, \mathrm{F})$, and tle $3 b(\mathrm{E})$, or combinations directed against tle $1+2 b(\mathrm{H})$, tle $1+3 b(\mathrm{I})$, tle $2 b+3 b(\mathrm{~J})$, and tle $1+2 b+3 b(\mathrm{~K})$. Single morpholino oligonucleotides were injected at a concentration of $600 \mu \mathrm{M}$ (C-F) and combinatorial injections $(\mathrm{H}-\mathrm{K})$ were performed using $300 \mu \mathrm{M}$ of each MO. Phenotypes of FITC-dextran positive embryos were observed after the beginning of eye pigmentation at stage 32 (B-F) and stage 28 (G-K). (B,G) Wild 
type control embryos were injected with $1 \times$ Yamamoto's and FITC-dextran. All embryos are shown in dorsal view with anterior at the top. Compared to the wild type controls $(B, G)$, morpholino injected embryos developed smaller eyes that were shifted towards the midline $(D, E, J, K)$ or cyclopic eyes $(C, H, I)$. In rare cases the eyes were lost entirely (E). (A) Phenotypes of FITC-positive embryos were categorized at stage 28-32 into weak and strong phenotypes. Weak phenotypes developed smaller eyes that were shifted towards the midline, whereas strong phenotypes showed cyclopic eyes. Eye-less phenotypes were included into the group of strong phenotypes. Abbreviations: $\mathrm{MO}$, morpholino oligonucleotide; WT, wild type. Scale bar 100 M. (PDF 10028 kb)

\section{Abbreviations}

aegPNA: N-[2-aminoethyl]-glycine PNA; AP: Anterior-posterior; Ipe: Lysinephosphonic-ester; MHB: Mid-hindbrain boundary; MO: Morpholino oligonucleotide; pePNA: Phosphonic ester PNA; PNA: Peptide Nucleic Acid; TML: Tri-methyl-Iysine

\section{Acknowledgements}

We thank Jochen Wittbrodt for the in situ probe directed against the bfl gene and Mary Wallis for critical reading of the manuscript.

\section{Funding}

The work was supported by the Austrian Research Promotion Agency (FFG, grant 836,444), the Austrian Science Fund (FWF, grant P19571-B11) and the City of Vienna (MA23 - project 18-18). The funding sources had no role in study design, collection, analysis or interpretation of data.

\section{Availability of data and materials}

The datasets used and analysed during the current study are available from the corresponding author on reasonable request.

\section{Authors' contributions}

$G D, S D, N A, B B$, and ER were responsible for experimental design, analysis, execution, and writing of the manuscript. MA, HB, BW and TL performed the PNA design and established and performed the chemical synthesis. TC was responsible for experimental design, analysis, writing, communication, and supervising the project. All authors read and approved the final manuscript.

\section{Ethics approval and consent to participate}

Not applicable.

\section{Consent for publication}

Not applicable.

\section{Competing interests}

$M A, H B, B W$ and $T L$ were full employees of the company ugichem $\mathrm{GmbH}$ that developed PNAs for therapeutic applications. Design and synthesis of the PNAs was performed at the ugichem $\mathrm{GmbH}$, which became insolvent 2015. All other authors declare no competing interests.

\section{Publisher's Note}

Springer Nature remains neutral with regard to jurisdictional claims in published maps and institutional affiliations.

\footnotetext{
Author details

'Department for Applied Life Sciences, University of Applied Sciences, FH Campus Wien, Helmut-Qualtinger-Gasse 2, A-1030 Vienna, Austria. ${ }^{2}$ Centre for Organismal Studies (COS), University of Heidelberg, Im Neuenheimer Feld 230, 69120 Heidelberg, Germany. ${ }^{3}$ Department of Hematology, Oncology, Immunology, Rheumatology and Pulmonology, University Hospital Tübingen, Otfried-Mueller-Strasse 10, 72076 Tübingen, Germany. ${ }^{4}$ Sandoz GmbH, Biochemiestraße 10, A-6250 Kundl, Austria. ${ }^{5}$ CAST Gründungszentrum GmbH, Wilhelm-Greil-Straße 15, A-6020 Innsbruck, Austria. ${ }^{6}$ UGISense AG, c/o Nordwind Capital GmbH, Residenzstrasse 18, 80333 München, Germany.
}

Received: 30 May 2017 Accepted: 11 December 2017

Published online: 09 January 2018

\section{References}

1. Summerton JE. Morpholinos and PNAs compared. Lett Pept Sci. 2003; 10:215-36

2. Summerton J. Morpholino antisense oligomers: the case for an RNase $\mathrm{H}$ independent structural type. Biochim. Biophys. Acta - gene Struct. Expr. 1999;1489:141-58.

3. Nielsen PE, Egholm M, Berg RH, Buchardt O. Sequence-selective recognition of DNA by strand displacement with a thymine-substituted polyamide. Science. 1991;254:1497-500.

4. Buchardt $\mathrm{O}$, Egholm M, Berg $\mathrm{RH}$, Nielsen PE. Peptide nucleic acids and their potential applications in biotechnology. Trends Biotechnol. 1993;11:384-6.

5. Egholm M, Buchardt O, Christensen L, Behrens C, Freier SM, Driver DA, et al PNA hybridizes to complementary oligonucleotides obeying the Watsoncrick hydrogen-bonding rules. Nature. 1993;365:566-8.

6. Ray A, Nordén B. Peptide nucleic acid (PNA): its medical and biotechnical applications and promise for the future. FASEB J. 2000;14:1041-60.

7. Nielsen PE, Haaima G, Lohse A, Buchardt O. Peptide nucleic acids(PNAs) containing thymine monomers derived from Chiral amino acids: hybridization and solubility properties OfD-lysine PNA. Angew Chemie Int Ed English. 1996;35:1939-42.

8. Urtishak KA, Choob M, Tian X, Sternheim N, Talbot WS, Wickstrom E, et al. Targeted gene knockdown in zebrafish using negatively charged peptide nucleic acid mimics. Dev Dyn. 2003;228:405-13.

9. Dorn S, Aghaallaei N, Jung G, Bajoghli B, Werner B, Bock H, et al. Side chain modified peptide nucleic acids (PNA) for knock-down of six3 in medaka embryos. BMC Biotechnol. 2012;12:50.

10. van de Wetering $M$, Oosterwegel M, Dooijes $D$, Clevers $H$. Identification and cloning of TCF-1, a T lymphocyte-specific transcription factor containing a sequence-specific HMG box. EMBO J. 1991;10:123-32.

11. Arce L, Yokoyama NN, Waterman ML. Diversity of LEF/TCF action in development and disease. Oncogene. 2006;25:7492-504.

12. Behrens J, von Kries JP, Kühl M, Bruhn L, Wedlich D, Grosschedl R, et al. Functional interaction of beta-catenin with the transcription factor LEF-1. Nature. 1996:382:638-42.

13. Arce L, Pate KT, Waterman ML. Groucho binds two conserved regions of LEF-1 for HDAC-dependent repression. BMC Cancer. 2009;9:159.

14. Kengaku M, Capdevila J, Rodriguez-Esteban C, De La Peña J, Johnson RL, Izpisúa Belmonte JC, et al. Distinct WNT pathways regulating AER formation and dorsoventral polarity in the chick limb bud. Science. 1998;280:1274-7.

15. Houston DW, Kofron M, Resnik E, Langland R, Destree O, Wylie C, et al. Repression of organizer genes in dorsal and ventral Xenopus cells mediated by maternal XTcf3. Development. 2002;129:4015-25.

16. Merrill BJ, Pasolli HA, Polak L, Rendl M, Garcia-Garcia MJ, Anderson KV, et al. Tcf3: a transcriptional regulator of axis induction in the early embryo. Development. 2004;131:263-74.

17. Cole MF, Johnstone SE, Newman JJ, Kagey MH, Young RA. Tcf3 is an integral component of the core regulatory circuitry of embryonic stem cells. Genes Dev. 2008;22:746-55.

18. Pereira L, Yi F, Merrill BJ. Repression of Nanog gene transcription by Tcf3 limits embryonic stem cell self-renewal. Mol Cell Biol. 2006;26:7479-91.

19. Kim CH, Oda T, Itoh M, Jiang D, Artinger KB, Chandrasekharappa SC, et al. Repressor activity of headless/Tcf3 is essential for vertebrate head formation. Nature. 2000:407:913-6.

20. Dorsky Rl, Itoh M, Moon RT, Chitnis A. Two tcf3 genes cooperate to pattern the zebrafish brain. Development. 2003;130:1937-47.

21. Brantjes H, Roose J, Van De Wetering M, Clevers H. All Tcf HMG box transcription factors interact with Groucho-related co-repressors. Nucleic Acids Res. 2001;29:1410-9.

22. Daniels DL, Weis WI. Beta-catenin directly displaces Groucho/TLE repressors from Tcf/Lef in Wnt-mediated transcription activation. Nat Struct Mol Biol. 2005;12:364-71.

23. Turki-Judeh W, Courey AJ. The Unconserved Groucho central region is essential for viability and modulates target gene specificity. PLOS One. 2012;7:e30610.

24. Roose J, Molenaar M, Peterson J, Hurenkamp J, Brantjes $H$, Moerer $\mathrm{P}$, et al. The Xenopus Wnt effector XTcf-3 interacts with Groucho-related transcriptional repressors. Nature. 1998;395:608-12. 
25. Mallo M, Franco del Amo F, Gridley T. Cloning and developmental expression of Grg, a mouse gene related to the groucho transcript of the drosophila enhancer of split complex. Mech Dev. 1993;42:67-76.

26. Schmidt CJ, Sladek TE. A rat homolog of the drosophila enhancer of split (groucho) locus lacking WD-40 repeats. J Biol Chem. 1993;268:25681-6.

27. Wang D, Manali D, Wang T, Bhat N, Hong N, Li Z, et al. Identification of pluripotency genes in the fish medaka. Int J Biol Sci. 2011;7:440-51.

28. Bajoghli B, Aghaallaei N, Heimbucher T, Czerny T. An artificial promoter construct for heat-inducible misexpression during fish embryogenesis. Dev Biol. 2004;271:416-30.

29. Brannon M, Brown JD, Bates R, Kimelman D, Moon RT. XCtBP is a XTcf-3 co-repressor with roles throughout Xenopus development. Development. 1999;126:3159-70.

30. Loosli F, Winkler S, Wittbrodt J. Six3 overexpression initiates the formation of ectopic retina. Genes Dev. 1999;13:649-54.

31. Mathers PH, Grinberg A, Mahon KA, Jamrich M. The Rx homeobox gene is essential for vertebrate eye development. Nature. 1997:387:603-7.

32. Bajoghli B, Aghaallaei N, Czerny T. Groucho corepressor proteins regulate otic vesicle outgrowth. Dev Dyn. 2005:233:760-71.

33. Lopez-Rios J, Tessmar K, Loosli F, Wittbrodt J, Bovolenta P. Six3 and Six6 activity is modulated by members of the groucho family. Development. 2003;130:185-95

34. Aghaallaei N, Bajoghli B, Walter I, Czerny T. Duplicated members of the Groucho/Tle gene family in fish. Dev Dyn. 2005;234:143-50.

35. Muhr J, Andersson E, Persson M, Jessell TM, Ericson J. Groucho-mediated transcriptional repression establishes progenitor cell pattern and neuronal fate in the ventral neural tube. Cell. 2001;104:861-73.

36. Chitnis $A B$, Itoh M. Exploring alternative models of rostral-caudal patterning in the zebrafish neurectoderm with computer simulations. Curr Opin Genet Dev. 2004;14:415-21.

37. Kiecker C, Niehrs C. A morphogen gradient of Wnt/beta-catenin signalling regulates anteroposterior neural patterning in Xenopus. Development. 2001; 128:4189-201.

38. Bajoghli B, Aghaallaei N, Jung G, Czerny T. Induction of otic structures by canonical Wnt signalling in medaka. Dev Genes Evol. 2009;219:391-8.

39. Bajoghli B, Aghaallaei N, Soroldoni D, Czerny T. The roles of Groucho/ Tle in left-right asymmetry and Kupffer's vesicle organogenesis. Dev Biol. 2007;303:347-61.

40. Miyasaka H, Choudhury BK, Hou EW, Li SS. Molecular cloning and expression of mouse and human CDNA encoding AES and ESG proteins with strong similarity to drosophila enhancer of split groucho protein. Eur J Biochem. 1993;216:343-52.

41. Chen G, Nguyen PH, Courey AJ. A role for Groucho tetramerization in transcriptional repression. Mol Cell Biol. 1998;18:7259-68.

42. Choi CY, Kim YH, Kwon HJ, Kim Y. The homeodomain protein NK-3 recruits Groucho and a histone deacetylase complex to repress transcription. J Biol Chem. 1999;274:33194-7.

43. Eberhard D, Jiménez G, Heavey B, Busslinger M. Transcriptional repression by Pax5 (BSAP) through interaction with corepressors of the Groucho family. EMBO J. 2000;19:2292-303.

44. Pinto M, Lobe CG. Products of the grg (Groucho-related gene) family can dimerize through the amino-terminal Q domain. J Biol Chem. 1996;271: 33026-31.

45. Yao J, Lai E, Stifani S. The winged-helix protein brain factor 1 interacts with groucho and hes proteins to repress transcription. Mol Cell Biol. 2001;21:1962-72.

46. Yu X, Li P, Roeder RG, Wang Z. Inhibition of androgen receptormediated transcription by amino-terminal enhancer of split. Mol Cell Biol. 2001;21:4614-25.

47. Zhang X, Chen H-M, Jaramillo E, Wang L, D'Mello SR. Histone deacetylaserelated protein inhibits AES-mediated neuronal cell death by direct interaction. J Neurosci Res. 2008:86:2423-31.

48. Palaparti A, Baratz A, Stifani S. The Groucho/transducin-like enhancer of split transcriptional repressors interact with the genetically defined amino-terminal silencing domain of histone H3. J Biol Chem. 1997; 272:26604-10.

49. Beagle B, Johnson GV. AES/GRG5: more than just a dominant-negative TLE/ GRG family member. Dev Dyn. 2010;239:2795-805.

50. Tetsuka T, Uranishi H, Imai H, Ono T, Sonta S, Takahashi N, et al. Inhibition of nuclear factor-kappaB-mediated transcription by association with the amino-terminal enhancer of split, a Groucho-related protein lacking WD40 repeats. J Biol Chem. 2000;275:4383-90.

51. Zhang Y, Gao S, Wang Z. Structural and functional analysis of aminoterminal enhancer of split in androgen-receptor-driven transcription. Biochem J. 2010;427:499-511.

52. Tsuji S, Hashimoto C. Choice of either beta-catenin or Groucho/TLE as a cofactor for Xtcf-3 determines dorsal-ventral cell fate of diencephalon during Xenopus development. Dev Genes Evol. 2005;215:275-84.

53. Jung G, Hug M, Halter C, Friesenhengst A, Walzer J, Czerny T. Diffusion of small molecules into medaka embryos improved by electroporation. BMC Biotechnol. 2013:13:53.

54. Boutagy J, Thomas R. Olefin synthesis with organic phosphonate carbanions. Chem Rev Am Chem Soc. 1974;74:87-99.

55. Cativiela C, Díaz-de-Villegas MD, Gálvez JA, Su G. Horner-WadsworthEmmons reaction for the synthesis of unusual alpha,beta-didehydroamino acids with a chiral axis. ARKIVOC. 2004;2003:59-66.

56. Wadsworth WS. Synthetic applications of Phosphoryl-stabilized anions. Org. React. Hoboken, NJ, USA: John Wiley \& Sons, Inc.; 1977. p. 73-253.

57. Burk MJ, Feaster JE, Nugent WA, Harlow RL. Preparation and use of C2symmetric bis(phospholanes): production of .Alpha.-amino acid derivatives via highly enantioselective hydrogenation reactions. J Am Chem Soc. 1993; 115:10125-38

58. Cobley CJ, Lennon IC, Praquin C, Zanotti-Gerosa A, Appell RB, Goralski CT, et al. Highly efficient asymmetric hydrogenation of 2-Methylenesuccinamic acid using a Rh-DuPHOS catalyst. Org Process Res Dev. 2003;7:407-11.

59. Lennon I, Pilkington C. The Application of AsymmetricHydrogenation for the Manufacture of Pharmaceutical Intermediates:The Need for Catalyst Diversity. Synthesis. 2003;11:1639-42.

60. Fink M, Flekna G, Ludwig A, Heimbucher T, Czerny T. Improved translation efficiency of injected mRNA during early embryonic development. Dev Dyn. 2006;235:3370-8.

61. Adams B, Dorfler P, Aguzzi A, Kozmik Z, Urbanek P, Maurer-Fogy l, et al. Pax5 encodes the transcription factor BSAP and is expressed in B lymphocytes, the developing CNS, and adult testis. Genes Dev. 1992;6:1589-607.

62. Pomerantz JL, Sharp PA, Pabo CO. Structure-based design of transcription factors. Science. 1995;267:93-6.

63. O'Shea EK, Lumb KJ, Kim PS. Peptide "Velcro": design of a heterodimeric coiled coil. Curr Biol. 1993;3:658-67.

64. Iwamatsu T. Stages of normal development in the medaka Oryzias Latipes. Mech Dev. 2004;121:605-18.

65. Loosli F, Koster RW, Carl M, Krone A, Wittbrodt J. Six3, a medaka homologue of the drosophila homeobox gene sine oculis is expressed in the anterior embryonic shield and the developing eye. Mech Dev. 1998;74:159-64.

66. Koster RW, Kuhnlein RP, Wittbrodt J. Ectopic Sox3 activity elicits sensory placode formation. Mech Dev. 2000;95:175-87.

67. Carl M, Wittbrodt J. Graded interference with FGF signalling reveals its dorsoventral asymmetry at the mid-hindbrain boundary. Development. 1999;126:5659-67.

68. Kitagawa D, Watanabe T, Saito K, Asaka S, Sasado T, Morinaga C, et al. Genetic dissection of the formation of the forebrain in Medaka, Oryzias Latipes. Mech Dev. 2004;121:673-85.

69. Vancha AR, Govindaraju S, Parsa KVL, Jasti M, Gonzalez-Garcia M, Ballestero RP, et al. Use of polyethyleneimine polymer in cell culture as attachment factor and lipofection enhancer. BMC Biotechnol. 2004;4:23.

\section{Submit your next manuscript to BioMed Central and we will help you at every step:}

- We accept pre-submission inquiries

- Our selector tool helps you to find the most relevant journal

- We provide round the clock customer support

- Convenient online submission

- Thorough peer review

- Inclusion in PubMed and all major indexing services

- Maximum visibility for your research

Submit your manuscript at www.biomedcentral.com/submit 